Research Article

\title{
Feature Clustering Analysis Using Reference Model towards Rolling Bearing Performance Degradation Assessment
}

\author{
Xiaoxi Ding $\mathbb{D},{ }^{1}$ Liming Wang $\mathbb{D},{ }^{1}$ Wenbin Huang, ${ }^{1}$ Qingbo $\mathrm{He}^{2}$ and Yimin Shao ${ }^{1}$ \\ ${ }^{1}$ State Key Laboratory of Mechanical Transmission, Chongqing University, Chongqing 400044, China \\ ${ }^{2}$ School of Mechanical and Power Engineering, Shanghai Jiao Tong University, Shanghai 200240, China \\ Correspondence should be addressed to Xiaoxi Ding; dxxu@cqu.edu.cn
}

Received 28 May 2019; Accepted 14 February 2020; Published 28 March 2020

Academic Editor: Giosuè Boscato

Copyright (c) 2020 Xiaoxi Ding et al. This is an open access article distributed under the Creative Commons Attribution License, which permits unrestricted use, distribution, and reproduction in any medium, provided the original work is properly cited.

The health monitoring and management have been accepted in modern industrial machinery for an intelligent industrial production. To timely and reliably assess the bearing performance degradation, a novel health monitoring method called feature clustering analysis (FCA) has been proposed in this study. Along with the working time going, this new monitored chart picked by FCA aims to describe the feature clustering distribution transition by a series of reference models. First, the data provided by the reference state (healthy data) and the one from the monitor state (monitor data) are fused together to construct a reference model, which is to explore the active role of healthy status and activate the difference between healthy status and unhealthy status. Manifold learning is later implemented to mine the discriminated features for good class-separable clustering measure. In this manner, heterogeneous information hidden in this reference model will appear once degradation happened. Finally, a clustering quantification factor, named as feature clustering indicator (FCI), is calculated to assess distribution evolution and migration of the monitor status as compared to the consistent healthy status. Furthermore, a single Gaussian model (SGM) based on these FCIs is used to provide a smooth estimate of the healthy condition level. The corresponding negative log likelihood probability (NLLP) and the fault occurrence alarm are developed for an accurate and reliable FCC. And it can well depict a comprehensibility of the real bearing performance degradation process for its whole life. Meanwhile, as compared to other health profiles based on the classical health indicators, the proposed FCC has provided a much more accurate degradation level and rather monotonic profile. The experimental results show the potential in machine health performance degradation assessment.

\section{Introduction}

Condition-based maintenance (CBM) has been widely accepted as an essential maintenance program in the modern industry. Based on the information acquired through condition monitoring, health monitoring and management are vital in ensuring safety, minimizing breakdowns, and reducing the production costs [1-3]. As an easily damageable but widely used part in rotating machinery, the bearing health condition needs to be timely and reliably identified [4-10], where the failure and loss can be effectively warned and prevented at an early level. Meanwhile, quite different from fault detection and fault diagnosis $[11,12]$, the rest crucial step for CBM is the analysis of the size or degradation level of a fault, where fault detection is to identify whether the equipment is or not in a normal status and fault diagnosis focuses on fault mode identification. And a more accurate health condition evaluation will bring much more benefits for decision-making process of CBM. As the mechanical vibration reveals the dynamic performance degradation of bearing health, a data-driven approach based on vibration analysis has been widely used in bearing health monitoring. In bearing health monitoring, health indicator (HI) is hoped to effectively degenerate following the bearing health condition with monotonicity overall, so that the full cycle life of the bearings can be accurately monitored with an early warning reached to trigger the slight degradation alarm, whereas there is a major challenge to construct a reliable and sensitive $\mathrm{HI}$ from the online or monitored vibration signals, which are always disturbed by the environmental noise and macrostructural 
disturbance. Therefore, monitoring HI construction from the nonlinear stochastic vibration signals is crucial in the application of bearing health monitoring.

In prognostic health management (PHM), one of the most important issues is to acquire the sensitive and representative features for an effective $\mathrm{HI}$ construction. Up to now, many advanced techniques have been working on the original features construction, which can be divided into three analysis domains: time-domain analysis, frequencydomain analysis, and time-frequency analysis. To further extract the sensitive features, feature selection (FS) [13-15] or feature extraction (FE) [16-22] techniques are often used as the preprocessing methods for bearing fault diagnosis and degradation assessment. The FS methods mainly focus on the selection of the most sensitive features based on the principle of the feature discrimination ability. By utilizing the internal liner/nonlinear characteristics in the raw features, the FE methods employ some optimization strategies to enhance the sensitivity of the extracted features. There are mainly two different kinds of FE methods: linear projection methods, such as principal component analysis (PCA) [13], linear discriminant analysis (LDA) [14], and locality preserving projections (LPP) [17] and nonlinear projection methods, including kernel PCA (KPCA), trace ratio linear discriminant analysis (TR-LDA) [20], local tangent space alignment (LTSA) [21], and locally linear embedding (LLE) [23]. Linear FE methods are practical and visualized but are not sufficient enough to extract the nonlinear structure in the nonstationarity data. Nevertheless, with the consideration of the local topology structure, the nonlinear FE methods can discover the intrinsic geometry structure of the raw data. It should be noted that, in the framework of the conventional health monitoring scheme, these mentioned original features or extracted features of the monitor dataset are always directly constructed and mapped based on the healthy dataset alone in the training process. Then, the new extracted features will be put into the next models for bearing fault diagnosis $[19,20]$, performance degradation assessment (PDA) [4-10], and residual useful life (RUL) prediction [23-25]. For example, Yu [7] obtained the feature mapping from the healthy data by LPP and put the mapped features of the monitor data into the Gaussian mixture model (GMM) for bearing performance degradation. Pan et al. [8] employed the extracted features from normal data to train a support vector data description (SVDD) model and health index based on general distance was designed. In the application of bearing health monitoring, Upadhyay and Rai [10] trained self-organizing map (SOM) from the original feature matrix of the normal data and used the support vector regression to assess the degradation.

Although these works have been succeeded in the application of bearing performance assessment, there still exist the following two characteristics that are not well considered. First, the reference model with the healthy data will play an important role in feature enhancement. According to the physical mechanism of the bearing failure, once a slight or severe fault happens, the surface of the monitored bearings will be worn or exfoliated. The corresponding periodic impact will produce transient components in the measured signal, which indicate the difference between the abnormal data and normal data. By introducing the normal data with abnormal data in a new reference model, it can be foreseen that the abnormal data will be easy to be classified from the normal data. Especially for health monitoring, the monitor data with a slight degradation occurrence can be clearly enhanced and thus timely detected. However, in the traditional framework, the normal data and the monitor data are always independent and the information will not reflect on the mappings/extracted features. Our recent work [17] has shown a significant difference between the feature space of healthy data and unhealthy data, which will effectively improve the clustering and distinguishability of multiclass features, including identification for some different degradation severities with similar feature distribution. In this manner, in our work in [18], we have tried to use reference features to evaluate the bearing performance degradation. Therefore, the reference model with healthy data will play an active role of healthy status and activate the difference between healthy status and unhealthy status.

Secondly, in the traditional monitoring systems, the extracted features are just constructed directly from the historical operation data under the assumption of healthy data. And from the principle of statistical quality control (SQC), it can be known that the feature distribution of the real-time monitor data will transfer from that of the healthy data along with the operating time going. According to the mentioned reference model, there is also a similar phenomenon that the relative feature clustering distribution of the two classes directly depicts the performance degradation level for each monitor data. When the monitor data has the same property with the healthy data, the feature clustering distribution of these two classes will be in coincidence or overlap with each other. Otherwise they will be divided into two populations, which mean that the monitor data is becoming damaged. Thus, the feature clustering distribution trend can be imported in bearing performance degradation, which can expose the distribution evolution and migration of the monitored reference model.

Motivated by these considerations, this study utilizes feature clustering distribution of the reference model with the healthy data to assess the performance degradation. Feature clustering analysis (FCA) is proposed instead of the complex mathematical monitoring models. First, a reference model is built by involving the same reference state (healthy data) with the monitored samples at each moment. Second the manifold feature extraction method is applied on each raw feature space for a two-class classification issue. As aforementioned, nonlinear FE methods show much better class-separable clustering measure for multiclass patterns. That is to say, their extracted features have better heterogeneity between different class patterns and show better homogeneity in the same class patterns. In this study, the nonlinear FE method, LLE, is implemented as a preprocessing method to deliver a better feature clustering distribution for the reference model. Then, a novel HI, feature clustering indicator (FCI), is proposed to quantitatively evaluate the class-separable clustering effect for each mapped reference model. In this manner, the performance 
degradation of the monitored bearings can be exposed by the clustering evolution of the monitor data as compared to the reference healthy data. Furthermore, to effectively and steadily monitor the current health and give an accurate fault occurrence alarm, the simple single Gaussian model (SGM) [7] is used to reach a smooth estimate of the healthy condition. In addition, the negative log likelihood probability (NLLP) and a fault occurrence alarm can be obtained. Finally, an improved SGM-based feature clustering chart (FCC) is developed for an accurate and reliable bearing health monitoring.

The rest of this paper is organized as follows: Section 2 presents the framework of the feature clustering system, where a new reference model-based FCA is proposed and its improved SGM-based FCC is developed. In Section 3, three whole-life bearings with comparisons are employed to verify the effectiveness of the proposed FCI. Finally, conclusions are drawn in Section 4.

\section{Feature Clustering Analysis}

In this section, a feature clustering analysis based on reference model is proposed for bearing health monitoring. Figure 1 illustrates the framework of FCA methodology. First, the critical issue is to choose the reference state for the reference model. In this study, the healthy data is regarded as the reference state, and the measured samples at monitor moment $t(t=1,2, \ldots, T)$ are combined with the reference state to construct the original reference matrix. Manifold learning is then implemented on the combined reference state to extract the meaningful features for a significant clustering distribution. In this manner, the characteristics of the monitored signal can be well highlighted as comparison with the same reference signal (healthy data). Thereafter, a discriminant clustering factor is calculated to evaluate the feature clustering of the mapped reference model at each monitor moment, so that the FCC will be achieved for online bearing health monitoring. Finally, SGM is further used to smoothly estimate the probability distribution evaluation and migration for the bearing health condition. Meanwhile, for a better illustration, Figure 2 draws the process of feature clustering analysis. Once the reference model was constructed with a baseline data, the manifold feature would be later mined via manifold learning, where a two-class feature space is provided to illustrate the degradation. A Gaussian model is further used to build a clustering chart for bearing PDA. Unlike the classical health monitoring scheme, with the same reference state, the feature clustering distribution of the reference model can expose the variety and heterogeneous information once a fault happened. The detailed information of the proposed feature clustering system, including general feature construction, reference modelling through manifold learning, FCA, and SGM-based FCC, is presented in the following.

2.1. General Feature Construction. In the process of FCA, the first operation is to construct a feature matrix for reference manifold. Lots of numerical features have been calculated to depict the equipment condition. In general, the time-domain statistical features are usually employed as the physical magnitude descriptors. This study calculates eight statistical features to build the original time-domain features [17], including the maximum peak value, kurtosis, absolute mean, crest factor, shape factor, root mean square (RMS), variance, and square root value. Simultaneously, as one of the famous feature generation techniques, wavelet transform (WT) has been widely used with its merit of multiresolution analysis. And by means of a series of low/high frequency pass filters, wavelet packet transform (WPT) can decompose a nonstationary signal into different frequency subspaces without energy loss. In this study, a three-level WPT with "db8" wavelet is employed to decompose the raw signal into eight frequency subbands. And the reconstructed wavelet coefficients of node $(j, n)$ can be expressed as $\left\{P_{j}^{n}(k), k=1,2, \ldots, N\right\}$, where $N$ is equal to the length of the input signal. The signal energy of each frequency subband is calculated as

$$
E_{3, i}=\sum_{k=1}^{N}\left[P_{j}^{n}(k)\right]^{2}
$$

Following this signal energy, the energy ratio in the third level to the total energy is reached as the final frequencydomain features as follows:

$$
r_{3, i}=\frac{e_{3, i}}{E_{t f}}, \text { where } E_{t f}=\sum_{i=0}^{7} e_{3, i} .
$$

The final features can be totally achieved as the feature set $\left\{f_{1}, f_{2}, \ldots, f_{16}\right\}$ in Table 1 . Therefore, a referenced feature matrix at monitor moment $t(t=1,2, \ldots, T)$ can be synthesized by combining the health feature set $\mathbf{H}=\left\{x_{1}\right.$, $\left.x_{2}, \ldots, x_{n 0}\right\}$ with sample number of $n_{0}$ and the corresponding feature set $\mathbf{N}_{t}=\left\{y_{1}, y_{2}, \ldots, y_{n t}\right\}$ with sample number of $n_{t}$ together. Thus, a reference feature model $\mathbf{M}_{t}=\left\{\mathbf{H}, \mathbf{N}_{t}\right\}$ with the sample number of $\left(n_{0}+n_{t}\right)$ is constructed for the following reference manifold feature space learning.

\subsection{Reference Model via Manifold Learning. As constructed} the reference model $\mathbf{M}_{t}=\left\{\mathbf{H}, \mathbf{N}_{t}\right\}$ for monitor moment $t$, this study implements nonlinear manifold learning method to pick out the inherent feature distribution. By means of manifold learning for this special reference mode, the inherent structure, which also stands for the natural difference between the monitored status and the referenced status, can be revealed. In this manner, the mined features of this reference model will have an outstanding class-separable clustering measure in a low-dimensional feature space. Here, a manifold learning algorithm, called LLE [23], is employed. By considering the neighbors of each sample with its corresponding weights, LLE can reconstruct the local expression combination in a global way. Therefore, the local geometry property, which means the neighborhood-preserving embedding hidden in the high-dimensional feature space, can be depicted in a low-dimension space. And the reference feature extraction based on LLE is simply described as follows. 


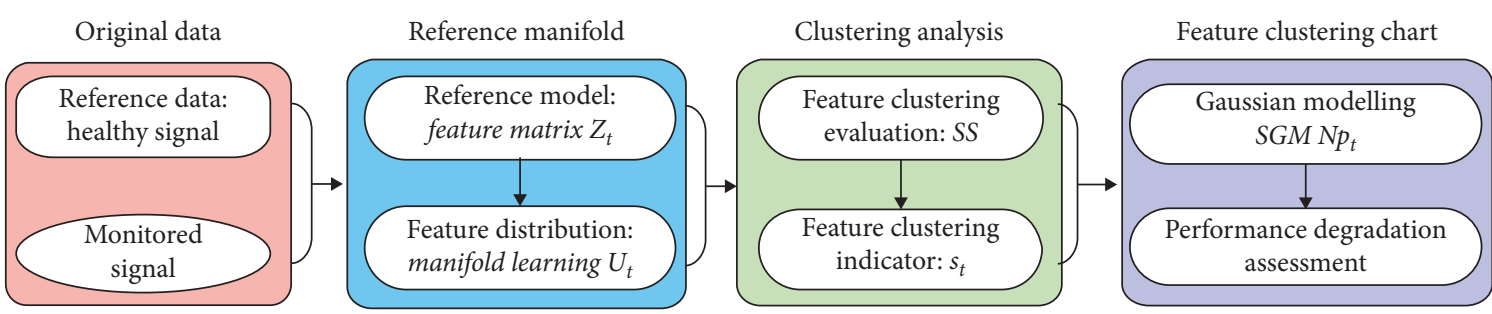

FIGURE 1: The framework of the feature clustering system for rolling bearing performance degradation assessment.
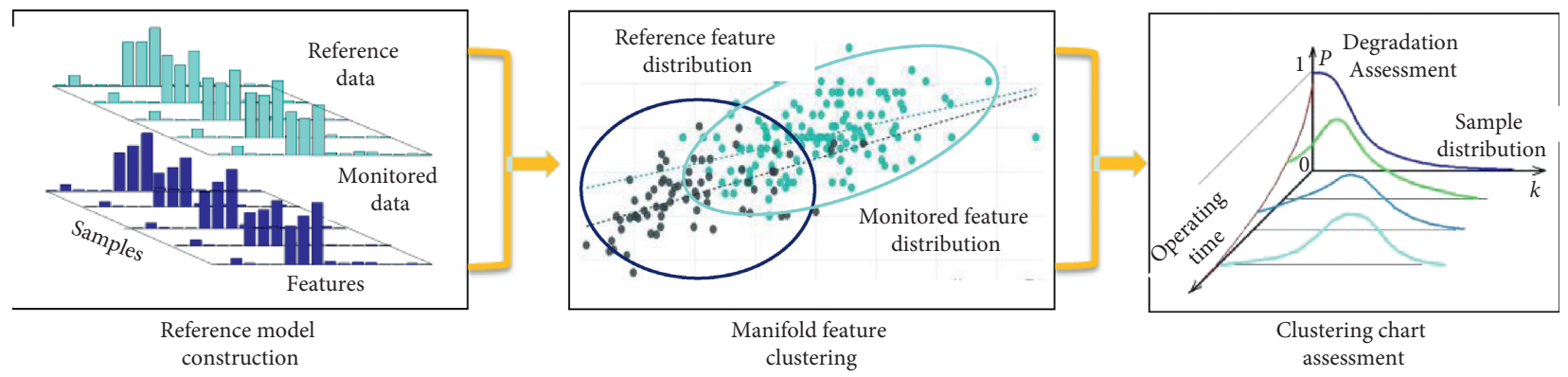

FIgURE 2: The illustration of feature clustering analysis.

TABLE 1: Original features.

\begin{tabular}{lccc}
\hline Number & Time domain & Number & Frequency domain \\
\hline$f_{1}$ & Maximum peak value & $f_{9}$ & $r_{30}$ \\
$f_{2}$ & Absolute mean & $f_{10}$ & $r_{31}$ \\
$f_{3}$ & Root mean square & $f_{11}$ & $r_{32}$ \\
$f_{4}$ & Variance & $f_{12}$ & $r_{33}$ \\
$f_{5}$ & Square root value & $f_{13}$ & $r_{34}$ \\
$f_{6}$ & Kurtosis & $f_{14}$ & $r_{35}$ \\
$f_{7}$ & Crest factor & $f_{15}$ & $r_{36}$ \\
$f_{8}$ & Shape factor & $f_{16}$ & $r_{37}$ \\
\hline
\end{tabular}

First, for the given high-dimensional feature set $\mathbf{M}_{t}=\{\mathbf{H}$, $\left.\mathbf{N}_{t}\right\}=\left\{z_{1}, z_{2}, \ldots, z_{n} \mid z_{i} \in R^{D}, i=1,2, \ldots, n ; n=n_{0}+n_{t} ; t=1\right.$, $2, \ldots, T\}$, the sensitive feature distribution is assumed to lie on or near a $d$-dimensional manifold space, where $d<<D$, $D=16$. To acquire the low-dimensional embedding for the reference mode, the LLE algorithm is conducted with three main steps as follows:

(1) Construct neighbors: select the $k$-nearest neighbors for each sample $z_{i}$ with the given neighbor number $k$.

(2) Reconstruct with local weights: achieve the best local reconstruction by minimizing the following error cost function:

$$
\varepsilon(W)=\sum_{i=1}^{n}\left\|z_{i}-\sum_{j=1}^{n} w_{i j} z_{j}\right\|^{2}
$$

where $w_{i j}$ is the weight between sample $z_{i}$ and sample $z_{j}$. If $z_{j}$ is not the neighbor of $z_{i}, w_{i j}=0$ and the local weights are subject to the constraint condition that $\sum_{j=1}^{n} w_{i j}=\sum_{l=1}^{k} w_{i}^{l}$.
(3) Embedded coordinates mapping: calculate the global internal coordinate $u_{i}$ by transforming the highdimensional vectors $z_{i}$ into a low-dimensional manifold space. Set $d$ as the final dimension of the output manifolds by minimizing

$$
\Phi(W)=\sum_{i=1}^{n}\left\|u_{i}-\sum_{j=1}^{n} w_{i j} u_{j}\right\|^{2}
$$

Satisfy the constraint condition $1 / N \sum_{i=1}^{n} u_{i} u s_{i}^{T}=\mathbf{I}_{d}$, $\sum_{i=1}^{n} u_{i}=\mathbf{0}$. By eigenvalue decomposition for the symmetrical coefficient matrix $\mathbf{M}=\left(\mathbf{I}_{N}-\mathbf{W}\right)^{T}\left(\mathbf{I}_{N}-\mathbf{W}\right)$, the bottom $d$ nonzero eigenvectors can be finally computed to form the embedding $\mathbf{U}_{t}=\left\{u_{i}\right\}_{i=1}^{n_{0}+n_{t}}(t=1,2, \ldots, T)$.

In this manner, the embedded coordinates $\mathbf{U}_{t}=\left\{\mathbf{P}, \mathbf{Q}_{t}\right\}$ can be captured by manifold learning, where $\mathbf{P}=$ $\left\{u_{1}, u_{2}, \ldots, u_{n_{0}}\right\}$ with size of $d \times n_{0}$ and $\mathbf{Q}_{t}=\left\{u_{n_{0+1}}, \ldots, u_{n_{0}+n_{t}}\right\}$ with size of $d \times n_{t}$ are the corresponding coordinates of the reference state $\mathbf{H}$ and the monitor data $\mathbf{N}_{t}$, respectively. The feature distribution of the embedded coordinates $\mathbf{U}_{t}$ based on reference model reflects the distribution 
variation between the current monitored data and the reference state. Along with the time $t$ going, this feature distribution will evolve into another status. Therefore, to depict the distribution variation, a discriminant clustering factor is further calculated from these coordinates $\mathbf{U}_{t}$ at time $t(t=1,2, \ldots, T)$, and the corresponding FCC is constructed to describe and assess the performance degradation as follows.

2.3. Feature Clustering Analysis. According to the reference model $\mathbf{U}_{t}=\left\{\mathbf{P}, \mathbf{Q}_{t}\right\}(t=1,2, \ldots, T)$, it can be easily foreseen that once a fault happened, there will be good classseparable clustering measure between the monitor data $\mathbf{Q}_{t}$ and the healthy data $\mathbf{P}$ due to their different distribution. For example, the feature RMS of a tested bearing on the whole life time is drawn in Figure 3. The dataset at moment point 1 is regarded as the reference state (here it is the healthy data). The other 9 runtime points along with the working time going, including 100, 300, 500, 680, 740, $780,860,920$, and 980 , are selected as the monitor moments (corresponding to the red dash-dotted lines). In addition, the number of samples in each moment is set to 20. Based on equations (3) and (4), a series of manifold coordinates from the reference models can be obtained for each runtime moment. Here, the corresponding feature clustering distribution of the reference feature sets is shown in Figure 4, respectively. As displayed in Figures 4(a)-4(c), it can be seen that there is no clear divisibility between the monitor data and the reference state except some deviation points, which indicates that these two datasets have the similar attributes and statuses. Furthermore, the feature distributions in Figures 4(d)4(g) have separation of trend with less samples overlapped at the moments $680,740,780,860$, and 920 . Note that the two categories are developing toward the opposite direction. Finally, the feature distributions are totally separated in Figure 4(i). Therefore, according to the bearing performance degradation and their corresponding feature clustering migration in Figure 4, it can be foreseen that the distribution of the reference model at each monitor moment depicts the trend of the bearing performance degradation along with the working time.

It is widely known that there are many feature extraction methods based on fisher classifier, which select the optimal mappings and sensitive features based on ratio of between-class distribution to within-class distribution in a supervised way. Especially, Lu et al. [19] proposed TR-LDA to find the maximal ratio of the between-class scatter $S_{b}$ to within-class scatter $S_{w}$ to make the surprised patterns in a well-distinguished scatter. Moreover, He et al. $[17,18,24]$ also calculated three parameters, including discriminant factor SS, between-class scatter $S_{b}$, and within-class scatter $S_{w}$, which have been successfully applied in feature evaluation and classification quality measurement. Here, to quantificationally evaluate the feature clustering distribution for bearing performance evolution, three parameters are calculated using the reference model $\mathbf{U}_{t}$ as follows:

$$
\begin{aligned}
& \mathrm{SS}=\frac{S_{b}}{S_{w}}, \\
& S_{b}=\operatorname{Tr}\left\{\sum_{j=1}^{c} n_{j}\left(\mu^{j}-\bar{\mu}\right)\left(\mu^{j}-\bar{\mu}\right)^{T}\right\}, \\
& S_{w}=\operatorname{Tr}\left\{\sum_{j=1}^{c} \sum_{u \in C_{j}}\left(u-\mu^{j}\right)\left(u-\mu^{j}\right)^{T}\right\}, \\
& \mu^{j}=\sum_{u \in C_{j}} \frac{u}{n_{j}}(j=1,2, \ldots, c), \quad \bar{\mu}=\sum_{j=1}^{c} \frac{\mu^{j}}{c},
\end{aligned}
$$

where $n_{j}$ is the sample number in the $j$ th class, $C_{j}$ is corresponding label set of the $j$ th class, and $c$ is the total number. The between-class scatter $S_{b}$ indicates the scattered level among different classes, while the within-class scatter $S_{w}$ describes the concentrated level in the same class. And the discriminant factor SS is a comprehensive indicator that inherits the property of between-class scatter and withinclass scatter. Basically, a larger $S_{b}$ indicates that the separation of classes is clearer and a smaller $S_{w}$ shows that the concentration of each class is relatively better, which together contributes a larger SS to reflect a better separable distribution of the monitored signal as compared to the healthy data. In this study, it should be noted that meanvariance standardization is also applied here to preprocess the feature vectors for a fair evaluation.

As listed in Table 2, the corresponding three indexes are calculated to evaluate the feature clustering distribution of each reference-model coordinate $\mathbf{U}_{t}(t=100,300,500,680$, $740,780,860,920,980)$. As operating time running, the between-class scatter $S_{b}$ shows an increasing trend with a decreasing trend for within-class scatter $S_{w}$. Thus, the comprehensive indicator SS gives a final degradation trend, which can effectively reveal the real-time operating condition of the monitor bearing health condition.

To further strengthen the sensitivity of comprehensive factor SS for precisely assessing bearing performance degradation and detecting the occurrence of the fault at an early stage, an exponentially weighted moving average (EWMA) [25] statistics is applied here. With the merits of small shifts detection for EWMA, it is very appropriate for SS-based EWMA statistic to form a monitor profile. Therefore, a novel $\mathrm{HI}$, called as feature clustering indicator (FCI), is proposed as follows:

$$
s_{t}=(1-\alpha) \mathrm{SS}_{t-1}+\alpha \mathrm{SS}_{t}, \quad \alpha \in[0,1),
$$

where a larger weight value of $\alpha$ allots more weight to current indicator $\mathrm{SS}_{t}$ and less weight to older indicator $\mathrm{SS}_{t-1}$. Generally, the value is taken between 0.01 and 0.25 [6]. In this study, the value of $\alpha$ is selected as 0.12 . According to the manifold feature distribution based on reference model, $s_{t}$ is calculated as a quantification index at each moment. This will lead a new profile to assess the performance degradation of the monitored bearing. 


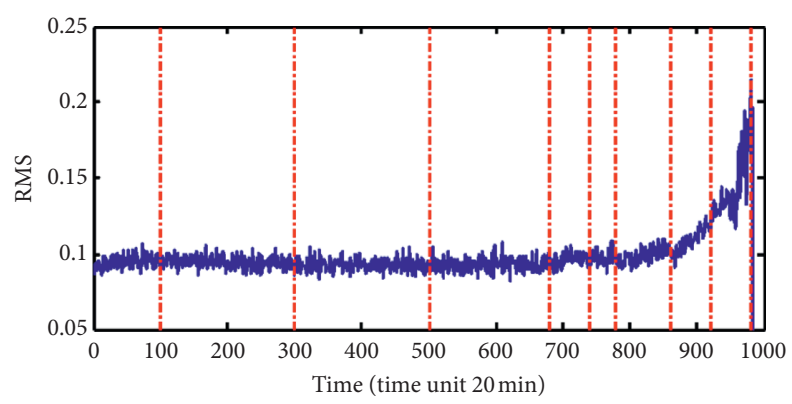

FIGURE 3: Bearing PDA based on RMS for the whole life time (the vertical red line corresponds to the monitor runtime moment).

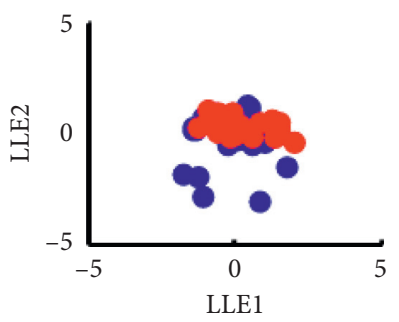

- Health data

- Monitored data

(a)

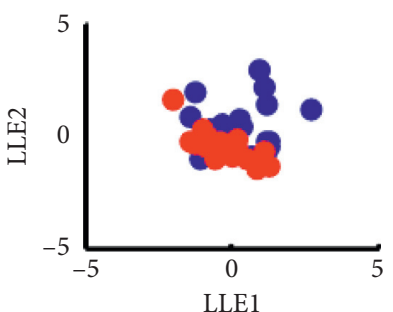

- Health data

Monitored data

(d)

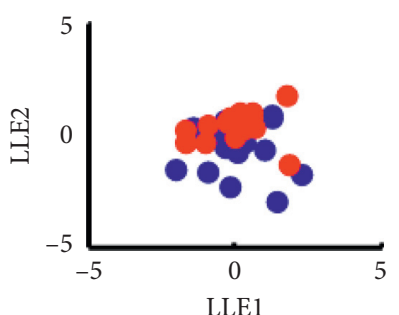

- Health data

- Monitored data

(g)

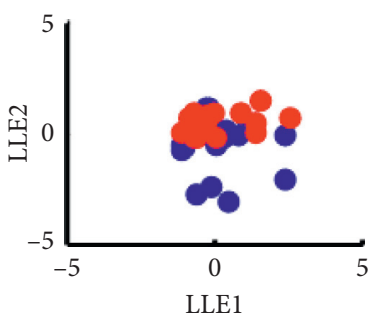

- Health data

- Monitored data

(b)

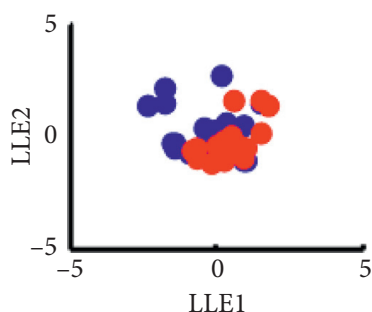

- Health data

- Monitored data

(e)

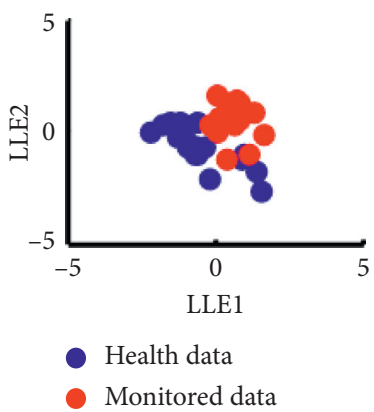

(h)

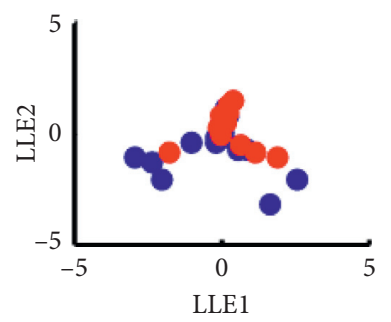

- Health data

- Monitored data

(c)

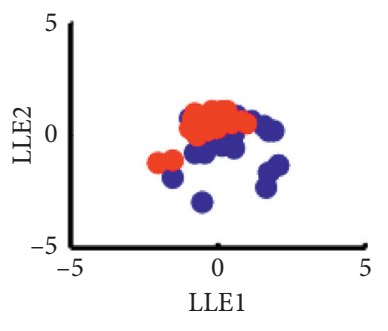

- Health data

- Monitored data

(f)

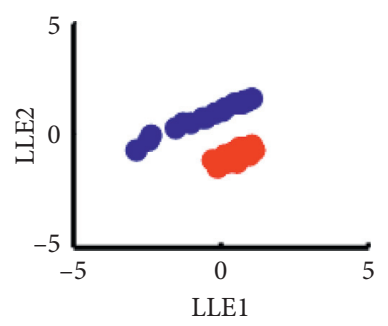

Health data

- Monitored data

(i)

Figure 4: Two-dimensional feature clustering distribution based on the reference model at different runtime moments: (a) 100, (b) 300, (c) 500, (d) 680, (e) 740, (f) 780, (g) 860, (h) 920, and (i) 980.

TAвLE 2: The feature clustering indicator for the monitor dataset at different runtime moments.

\begin{tabular}{llllllllll}
\hline Runtime moment & 100 & 300 & 500 & 680 & 740 & 780 & 860 & 920 & 980 \\
\hline Sb & 5.77 & 7.30 & 8.51 & 10.9 & 12.0 & 12.5 & 16.2 & 18.4 & 19.3 \\
Sw & 4.59 & 4.51 & 4.45 & 4.33 & 4.27 & 4.25 & 4.06 & 3.96 & 3.91 \\
SS & $\mathbf{1 . 2 6}$ & $\mathbf{1 . 6 2}$ & $\mathbf{1 . 9 1}$ & $\mathbf{2 . 5 3}$ & $\mathbf{2 . 8 1}$ & $\mathbf{2 . 9 5}$ & $\mathbf{3 . 9 9}$ & $\mathbf{4 . 6 5}$ & $\mathbf{4 . 9 3}$ \\
\hline
\end{tabular}


2.4. SGM-Based Feature Clustering Chart. Since the operating environment is easily interfered and there is not much priori information about the defect progression, it is not scientific and effective to steadily monitor current health status without considering the historical information. In health monitoring of the critical machine components, a universal process control can be learned from the large amount of healthy data. Under the assumption that only healthy data are accessible and they will meet with the normal distribution, SGM is further imported to model the data with a Gaussian distribution. Actually, another improved Gaussian model (Gaussian mixed model, named as GMM) [8] can be also employed to describe the healthy data with complicated distribution. It is easily seen that the SGM is a special case of GMM with the number of the mixed models being one. With the calculation of FCI, SGM is later used to describe the distribution of these FCIs and provide a smooth estimate of the health condition. Meanwhile, a new input data will deviate from this healthy distribution once the bearing status begins to degenerate. In these manners, through these FCIs, the SGM-based probability estimate can be further employed for PDA, which is named as SGM-based FCC.

In this SGM-based monitoring system, the distribution of the calculated FCIs via feature clustering analysis can be modeled by a single normal probability distribution function $(\mathrm{PDF})$, which is defined as

$$
p\left(\frac{s}{u, \delta}\right)=\frac{1}{\sqrt{2 \pi \delta^{2}}} \exp \left[-\frac{(s-u)^{2}}{2 \delta^{2}}\right],
$$

where $s$ is the calculated one-dimensional FCI and $u$ and $\delta$ are the mean and variance of the distribution $N(u, \delta)$, which is denoted as parameter $\theta(u, \delta)$. For a training set $s=\left[s_{1}, s_{2}\right.$, $\left.\ldots, s_{m}\right]$ with $m$ samples of variable HIs, the optimum parameter $\theta$ can be learned by the normal parameter estimation function with given confidence intervals. Once SGM is learned from those FCIs, the corresponding PDF is further employed to assess the bearing health condition online. For a new input, the unconditional probability density $p(s / \theta)$ indicates the level of the input following the probability distribution of the healthy state. The value $p$ of an input from the degenerated bearing will be beyond the value region of the healthy data (out of control from a threshold). Therefore, the value $p$ can be taken as an effective monitored indicator for assessing the bearing performance. Generally, to improve its legibility and intelligibility, negative $\log$ likelihood probability (NLLP) of the density value $p$ is also calculated as the monitor $\mathrm{HI}$ in this study:

$$
N p_{t}=-\log \left(p\left(\frac{s_{t}}{\theta}\right)\right), \quad t=1,2, \ldots, T .
$$

In statistical quality control (SQC), a threshold is needed to provide an alarm for the beginning of bearing performance degradation at the slight level. Kernel density estimation (KDE) [26] is used to define the alerting operating line. In this study, using kernel-smoothing method, the density estimate can be calculated by the function "ksdensity" of the Matlab software. With the confidence level 99.5\% (that means the false alarm rate is $0.5 \%$ for healthy data), the up/low warning line (UWL/LWL as the threshold) can be further obtained to confirm the confidence bound/threshold for indicator $N p$.

2.5. Monotonicity Evaluation for Feature Clustering Chart. In the application of bearing health monitoring, for an accurate detection and precise monitoring, the monotonicity of the degradation profile is also very important for system monitoring and maintenance. According to the mechanism of bearing health degradation, an HI with monotonicity over time is critical to machine PDA. In general, spearman coefficient (SC) [27] was applied to evaluate the monotonic relationship between two stochastic sequences. This study employs this coefficient to assess the monotonicity of the indicator over the operating time. The SC can be described by the following monotonic function:

$$
C_{s}=\left|1-\frac{6 \sum D^{2}}{N\left(N^{2}-1\right)}\right|,
$$

where $C_{s}$ is the spearman rank correlation coefficient (SRCC), $D$ is the difference between the two given sequences, and $N$ is the length of the sequences. Generally, a higher SC illustrates a strong monotonic relationship between the HI and the operating time of bearing performance degradation. Therefore, the effectiveness of the proposed FCC for rolling bearing health PDA can be characterized by SC value.

\section{Experimental Verification}

3.1. Experimental Data Description. To verify the effectiveness of the proposed FCA using reference model, a case of bearing running to failure experiment is tested in this study. The experimental data was downloaded from Prognostics Center Excellence (PCE) [28]. As plotted in Figure 5, there were totally four test Rexnord ZA-2115 double row bearings on the shaft of the bearing test rig. A radial load of $6000 \mathrm{lbs}$ was added to the shaft and bearing by a spring mechanism, where the rotation speed is constantly kept at $2000 \mathrm{rpm}$. Test would stop once the accumulated debris adhered to the magnetic plug exceeded a certain level and caused a switch to close. The data length was 20480 points with the sampling frequency rate of $20 \mathrm{kHz}$. In this study, bearing 2 (2\#) and bearing 4 (4\#) of testing 2 with outer-race failure and bearing 3 (3\#) of testing 1 with inner-race failure were used to illustrate the performance of the proposed FCC and SGMbased FCC.

3.2. Feature Clustering Results. In the application of rolling bearing health prognosis and fault diagnosis, one important issue is early detection and quantitative analysis for rolling bearing health PDA. Actually, it is seen that there will be no prior information about the defect severity except for the healthy state. Therefore, the main idea of the proposed FCA is to import the healthy data as the reference state and the defect severity of the monitor data at each moment can be exposed via the feature clustering distribution based on the reference model. Here, the healthy data size $n_{0}$ and the monitor data size $n_{t}$ are both set to be 20 with the sample length of 1024. The total number of each reference model is 


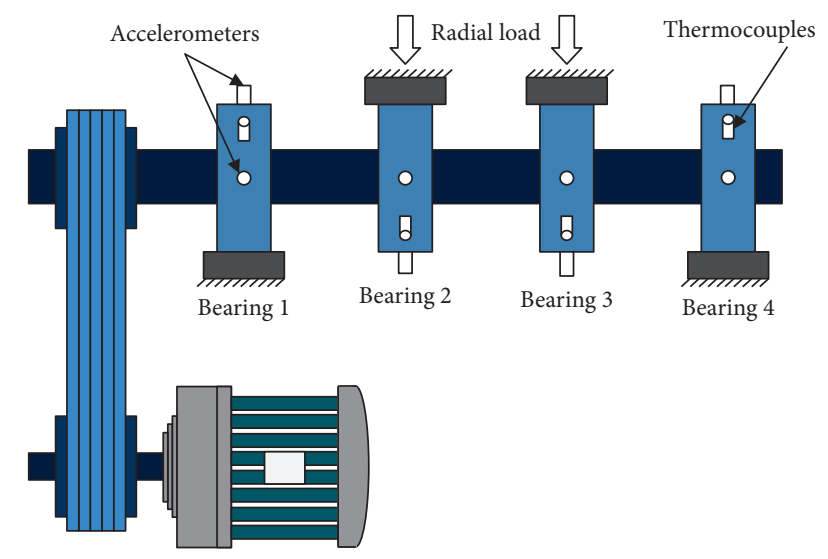

Figure 5: Bearing test rig.

40 and then LLE algorithm is implemented based on these 16-dimensional matrices combined with healthy data and the monitor data together. The dimension $d$ is kept as 5 . Thus, a reference matrix of size $5 \times 40$ is achieved at the monitoring time $t(t=1,2, \ldots, T)$. It should be noted that the same healthy dataset is employed in whole monitoring process. The FCI is later calculated from these mapped coordinates to assess the performance degradation of the monitored bearing at each corresponding moment.

Compared with the proposed FCI, three typical HIs, including RMS $\left(f_{3}\right)$, Kurtosis $\left(f_{6}\right)$, and the frequency energy ratio $\left(f_{9}\right)$, are first calculated for three tested bearings on their whole life, which are plotted in Figures 6(a)-6(c), respectively. It can be easily seen that there is a long stable interval in the RMS-based HI profile for the bearing on its whole life while the period of failure development is relatively short. And the other HIs $f_{3}, f_{6}$, and $f_{9}$ have inconsistent degradation patterns for the tested bearings under the same working conditions. Simultaneously, these HI profiles show no clear monotonicity along with the bearing performance degradation over working time. Moreover, it is not easy to further confirm their health degradation levels, such as the accurate failure occurrence. According to the conventional HI construction way, another widely used FE method PCA is additionally employed on the original healthy feature sets and the corresponding 1st principal component (PC) is calculated as the final $\mathrm{HI}$ for each monitor moment. And this PCA-based PC profile is displayed as Figure 6(d), which shows the same shortcomings as illustrated in the original HI profiles. It should be noted that these four HI profiles are subjected to traditional HI construction approaches (not the reference model). This means that there is a demand for a prognostic approach, which can be able to identify the slight degradation at the early level, so that effective maintenance can be taken to rebuild its operating health condition. It is hoped to get a monotonic HI profile to accurately detect the bearing health condition degradation statuses.

The proposed FCC based on reference model is different from the above traditional FE deterministic models. As displayed in Figure 7, FCI profiles for these three bearings reveal a much more stable and clearer degradation trend with good monotonicity, and the corresponding degradation levels are obviously exposed and quite clear in the their full cycle-life process of health performance degradation, which will bring benefits in the application of bearing health monitoring. Therefore, compared to other HI profiles, four conclusions can be drawn as follows: (1) a phased degradation can be obviously found in the bearing degradation processes, including healthy status, slight degradation status, and severe degradation status; (2) a significant and sharp change in the FCC would occur once the monitored bearing degenerates from healthy status to slight degradation as drawn in the blocks of Figure 7. Generally, these changing points in the short period can be used to timely and accurately verify the occurrence of faults, which are beneficial to identifying the degradation at an early level. In this manner, an early alarm can be sent and effective maintenances can be arranged to restore bearing health. Simultaneously, there is a duration period from slight degradation to severe degradation, so we can accurately master the bearing conditions before effective maintenance is taken; (3) the proposed HI profiles show an outstanding monotonicity with the time, which is critical for effective monitoring of bearing health monitoring and accurate prediction of bearing residual life; (4) a particularly interesting phenomenon can show that there is a stabilized level from the slight degradation status to the severe degradation status for bearing 2 and bearing 3, and at the same level, the rising trend of the performance degradation in bearing 4 slows down. In these special periods, their FCIs will not rise or fall too much and stay in a relatively stable range. Actually, these results are well consistent with the physical mechanism of bearing wear. The local contact surface of the bearings will be hardened again by the energy in the premature wear process. And this will effectively improve the abrasion performance of the bearings. Thus, after the bearings have been into the slight degradation level, the degradation trend will slow down or just enter into a stable area. As the working time going, the hardened surface of the bearing will be worn and destroyed at last. Under this operation, the bearing will be continuously worn in an irreversible process until failure. Therefore, after these stable degradation areas, the bearing will rush into another rapid degradation area and the degradation speed will be faster than the early degradation. The above analysis means that the actual bearing health condition is in phased degradation. According to the fact that the degradation speeds are mutative in the whole bearing life, many researchers used the piecewise prediction models for health monitoring and residual life prediction. This further strengthens the rationality and reliability of our proposed FCC as it clearly reveals different levels of the health degradation. Therefore, it is meaningful that the FCI provides a comprehensible $\mathrm{HI}$ in the real-word application of bearing health monitoring.

Meanwhile, another traditional statistical $T^{2}$ chart is also obtained from these reference model based on LLE. The result of the $T^{2}$ chart is shown in Figure 6(e). It can be found that there is a long stable level along the time for the healthy status, and a sharp change appears along with the fault happened. However, there is an obvious jitter in the period from slight degradation to severe degradation. Therefore, the 


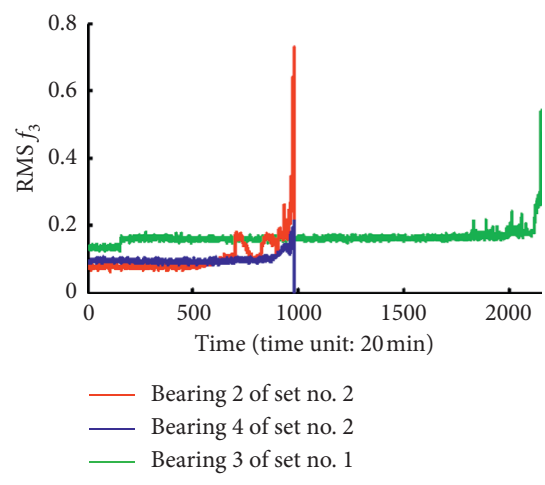

(a)

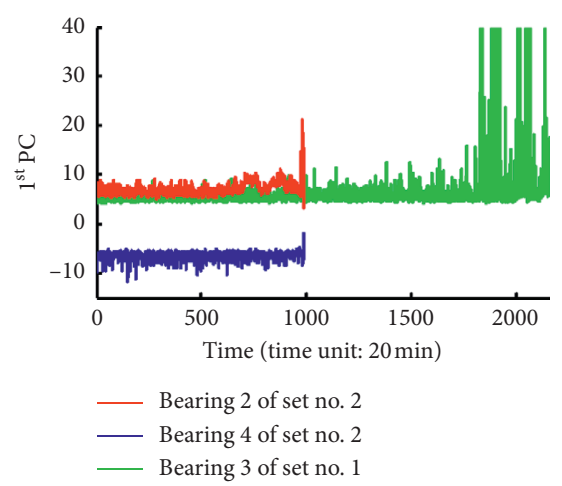

(d)

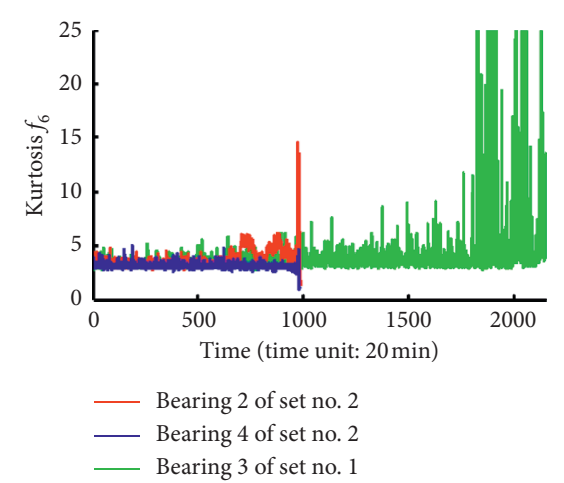

(b)

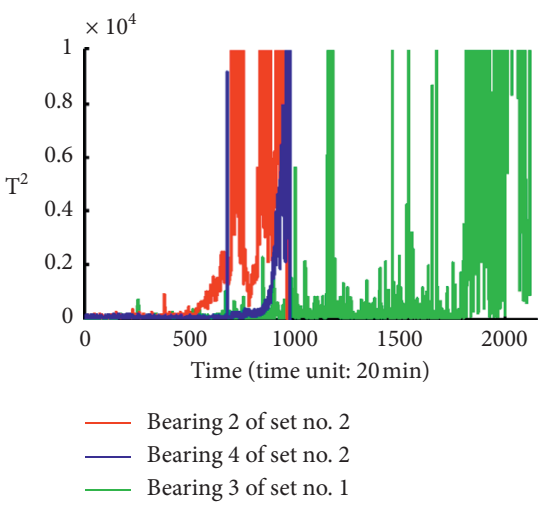

(e)

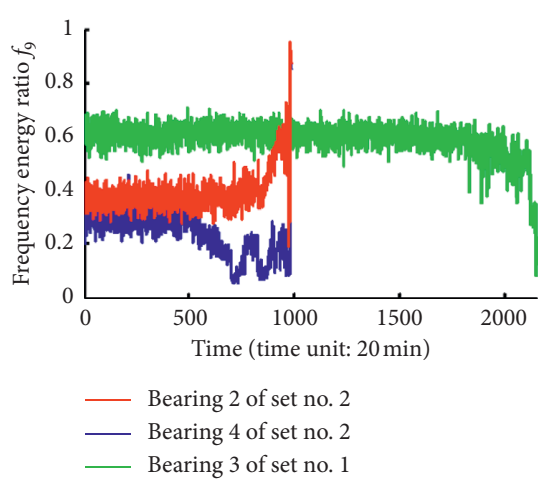

(c)

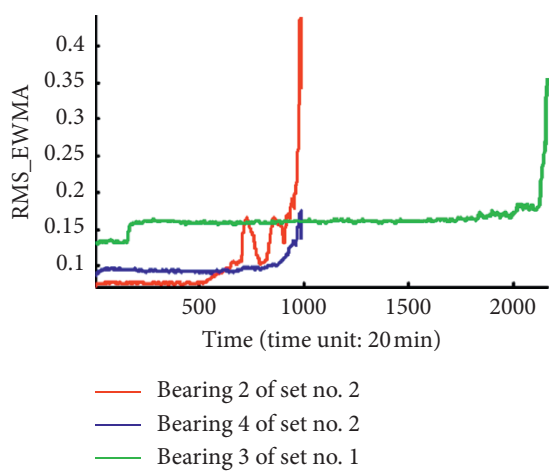

(f)

FIgURE 6: The PDA results for the full cycle life of the tested bearings: (a) the feature RMS $\left(f_{3}\right)$, (b) the feature kurtosis $\left(f_{6}\right)$, (c) the feature frequency energy ratio $\left(f_{9}\right),(\mathrm{d})$ the $1^{\text {st }}$ PC feature, (e) the $T^{2}$ chart, and (f) the indicator RMS_EWMA (RE).

$T^{2}$ chart profiles have no favorable monotonicity, which is not beneficial to effectively online monitoring. In the calculation of FCI from the discriminant factor, the EWMA technique is used to detect the small shifts of the clustering quantitation factor. Therefore, for a fair comparison, another HI named as RE, which is the feature RMS $\left(f_{3}\right)$ based on EWMA technique with the same parameter, is also calculated as shown in Figure 6(f). The smoothness and continuity of the monitor profiles become much better than those in Figure 6(a). Nevertheless, the slight degradation occurrences are not effectively enhanced with obvious jumping signature and there also exist severe jitter in the degradation profiles. According to these results, the proposed FCI profile calculated from reference model has competitive merits and a good potential in bearing health monitoring.

3.3. Monotonic Comparison. To further quantify the effectiveness of the proposed FCI chart, the SCs are calculated by (7) for those HI profiles. As shown in Table 3 and Figure 8, the HIs based on the reference model with manifold learning, including $T^{2}, \mathrm{RE}$ and $s$, have relatively better monotonic profiles for bearing performance degradation. This further exposes that the proposed reference model based on manifold learning shows a good potential in extracting and enhancing the discriminative characteristics between the reference state and the monitor data. Meanwhile, the SCs for the proposed FCI charts of bearing 2 and bearing 3 have bigger values than the ones of $T^{2}$ chart, which follow the same distributions as illustrated in Figure 6(e) and Figure 7 for bearing 2 and bearing 3. And it can be seen that the SC for the FCI chart of bearing 4 with value 0.45 is bit smaller than the one for the $T^{2}$ chart with value 0.50 . However, for $\mathrm{HI}$ profiles of bearing 4 , the FCI chart in Figure 7 shows a much better monotonicity overall with clearer degradation levels while there is a big variance in the severe degradation of $T^{2}$ chart as shown in Figure 6(e). Since SC is an overall evaluation coefficient for the whole-life bearing health degradation and there is a long stable period for the health status for $T^{2}$ chart of bearing 4 , the SC of $T^{2}$ chart will go up in some cases. In general, this SC can illustrate the good monotonicity of the proposed feature clustering profiles. Therefore, the proposed FCI chart from reference model based on manifold learning is beneficial to bearing health monitoring.

3.4. Assessment Performance Comparison. In this study, for the purpose of accurate and reliable health monitoring, SGM is later employed to give a probability of the monitor data based on a smooth estimate of the FCIs of the healthy data. These HIs of the healthy dataset (i.e., 300 samples) for each bearing are used to train the SGM model and the corresponding NLLP $N p$ are calculated for each monitored signal. Meanwhile, the threshold for the fault occurrence alarm is determined by the KDE tool. Therefore, the proposed 


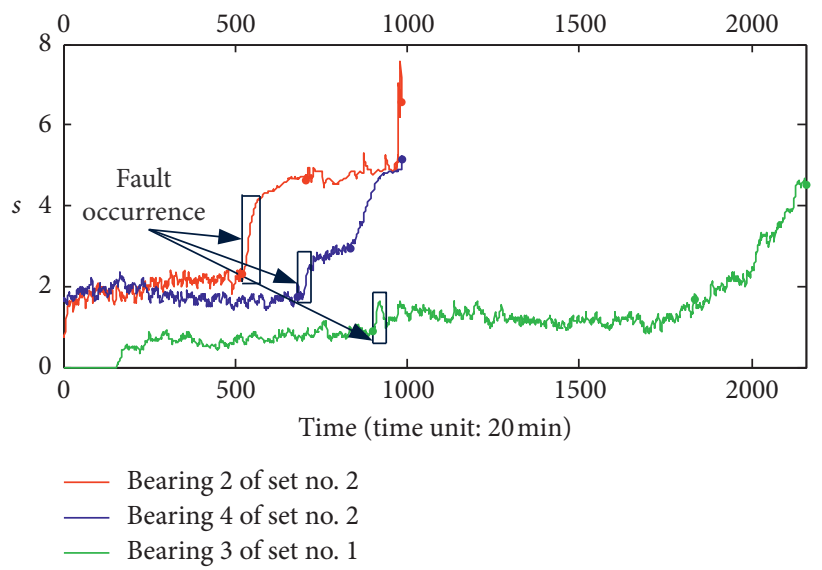

Figure 7: The cycle-life bearing PDA results of the proposed feature clustering chart based on the reference model.

TABLE 3: Spearman coefficients of different HIs for different bearing failure on its whole life time.

\begin{tabular}{lcccccccc}
\hline HI & $f_{3}$ & $f_{6}$ & $f_{9}$ & $1^{\text {st }}$ PC & $T^{2}$ & RE & SS \\
\hline 2\# outer-race & 0.81 & 0.54 & 0.75 & 0.44 & 0.85 & 0.84 & 0.86 \\
4\# outer-race & 0.41 & 0.14 & 0.41 & 0.12 & 0.50 & 0.42 & 0.52 & 0.96 \\
3\# inner-race & 0.59 & 0.34 & 0.39 & 0.33 & 0.79 & 0.81 & 0.74 & 0.88 \\
\hline
\end{tabular}

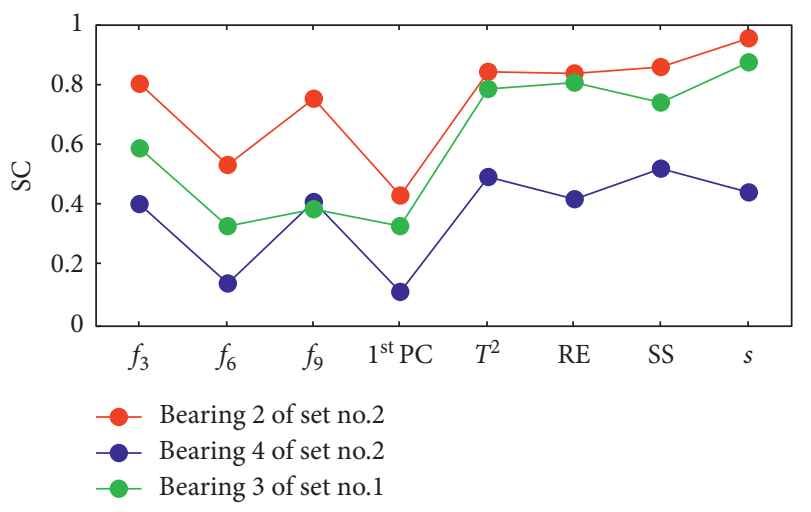

FIGURE 8: Spearman coefficient comparison of different HIs for different bearing failure on its whole life time.

SGM-based FCC can be used to evaluate the monitored bearing performance degradation. The profiles with the values NLLP Np for the full cycle life are drawn in Figure 9. In comparison with aforementioned HIs, the proposed FCCs also well inherit the merits of the FCIs and have a more stable and clearer trend of the performance degradation in the whole cycle life. Specifically, as found in the local enlargement of the assessment results in Figure 9, an early degradation alarm is activated by the proposed chart for each bearing, and the $N p$ would cross the given threshold once a fault happened. The trigger values for three bearings as shown in Figure 8 are 502, 709, and 902, respectively. Moreover, using locality preserving projections (LPP) and GMM, Yu [7] also proposed a health assessment indication to test these whole-life bearings, and the trigger values are 651,826 , and 1664, respectively. In comparison, it can be easily found that the SGM-based FCC profiles give much earlier and more distinct trigger values. Meanwhile, for bearing 3 of testing 1, the regions between trigger value 902 and trigger value 1664 just correspond to the stabilized level as mentioned in Figure 7, nearly from time point 900 to time point 1750. This means that the proposed SGM-based FCC delivers a much more accurate PDA and reveals the actual bearing health condition in phased degradation, which was easily ignored in other degradation profiles.

For a sound exposition, the corresponding fault information of these signals at the critical points is detected and verified. First, the time-domain signal at the breakdown values of the whole-life bearings $2 \#, 4 \#$, and $3 \#$ is, respectively, analyzed, and their envelope spectrums at point 960 , 960, and 2000 are drawn in Figures 10(c), 11(c), and 12(c). It can be easily found the corresponding fault frequencies are 


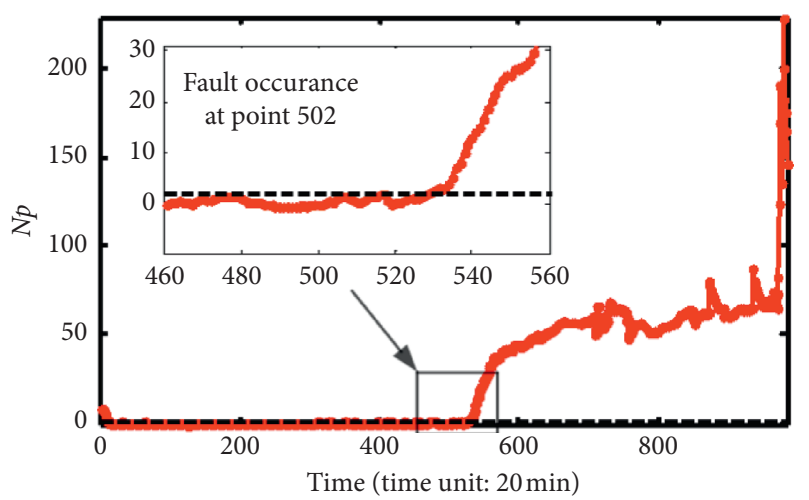

(a)

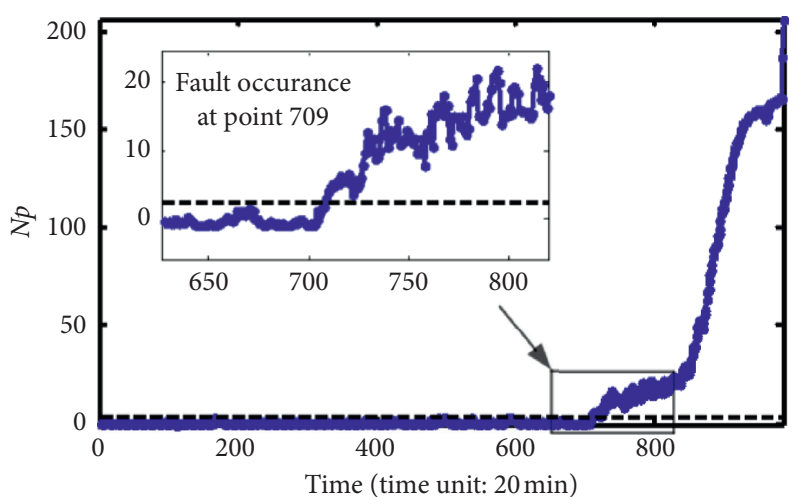

(b)

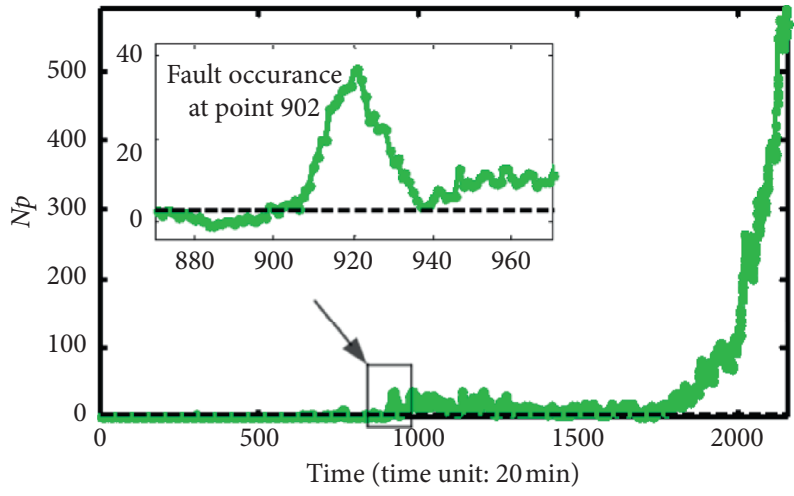

(c)

FIGURE 9: The assessment results and its local enlargement based on the proposed SGM-based FCC profile for the full cycle life of the three tested bearings: (a) bearing 2 of testing 2, (b) bearing 4 of testing 2, and (c) bearing 3 of testing 1 (the black dotted line is the corresponding threshold calculated by the KDE method).

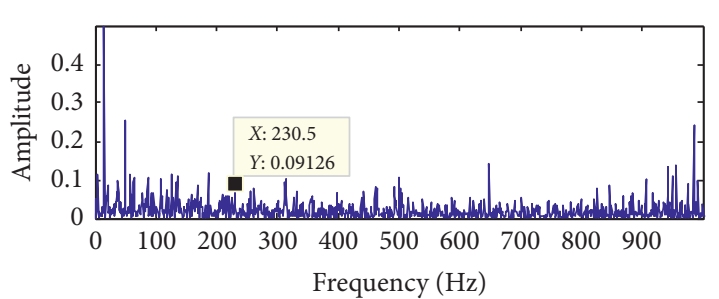

(a)

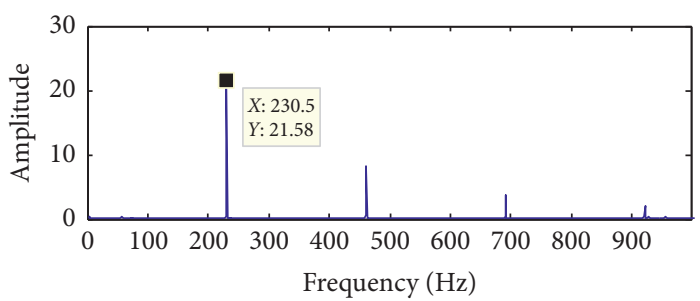

(b)

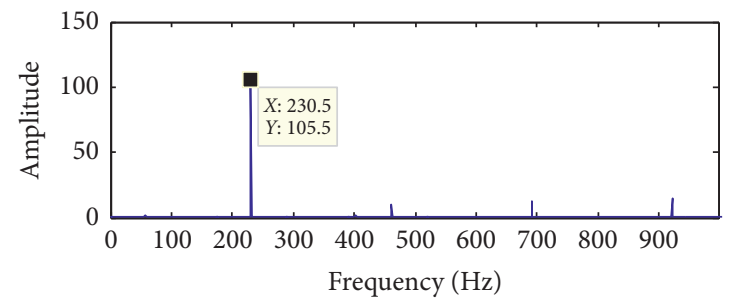

(c)

Figure 10: The envelope spectrums in the test for bearing 2\# of testing 2 at different operating moments: (a) the trigger value of the proposed method at point 502, (b) the trigger value of the compared method at point 651 , and (c) the breakdown value of the whole-life bearing at point 960 .

$230.5,63.48$, and $57.62 \mathrm{~Hz}$, which shows the maximum amplitudes. Meanwhile, the signals at the trigger values of the proposed FCC and the compared method [7] are also transformed. As demonstrated in Figures 10-12, it can be seen that, compared to the ones mentioned in [6], the fault information exposed by the proposed FCC has already 


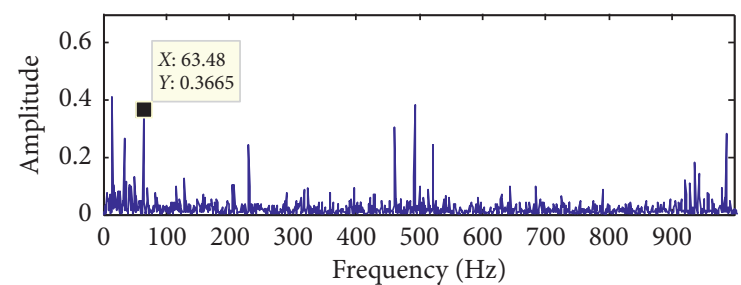

(a)

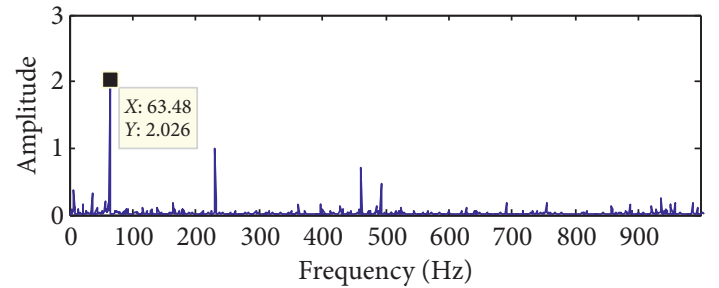

(b)

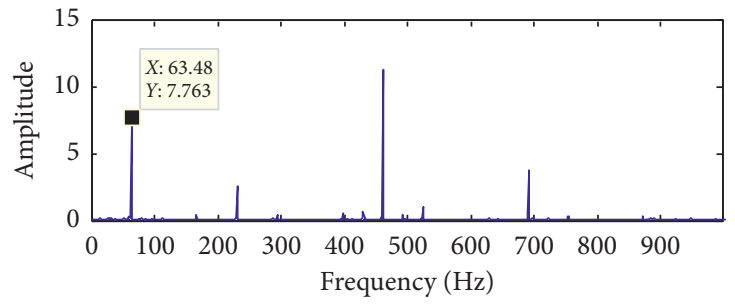

(c)

FIGURE 11: The envelope spectrums in the test for bearing 4\# of testing 2 at different operating moments: (a) the trigger value of the proposed method at point 709, (b) the trigger value of the compared method at point 826 , and (c) the breakdown value of the whole-life bearing at point 960 .

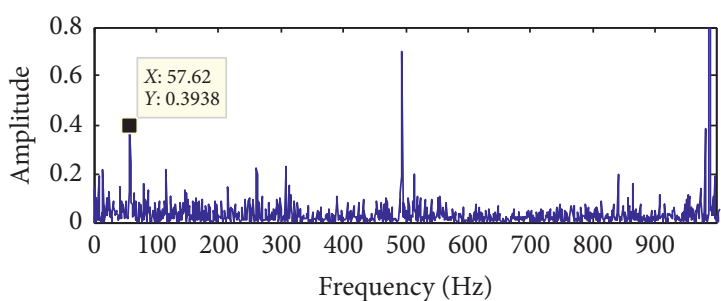

(a)

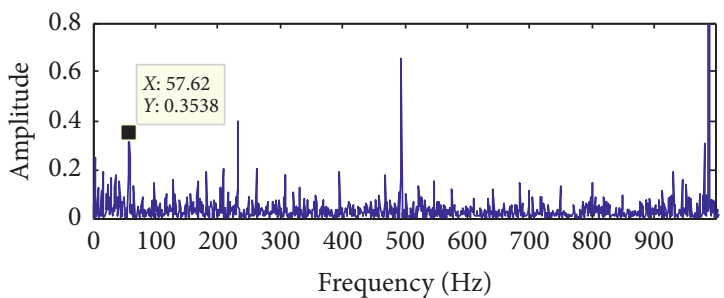

(b)

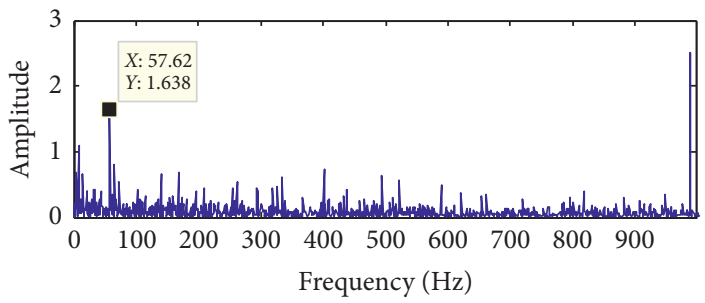

(c)

FIGURE 12: The envelope spectrums in the test for bearing 3\# of testing 1 at different operating moments: (a) the trigger value of the proposed method at point 902, (b) the trigger value of the compared method at point 1664, and (c) the breakdown value of the whole-life bearing at point 2000.

appeared in the spectrums with relatively small amplitude values, which indicate that the proposed FCC can research a much earlier failure warning for a much accurate monitoring performance. According to these experiments and comparisons, the proposed SGM-based FCC is effective to be employed in the application of rolling bearing PDA.

\section{Conclusions}

In this paper, a novel health monitoring scheme is proposed to assess the bearing performance degradation, which utilizes the feature clustering distribution of a reference model to depict the bearing health condition. Based on the reference model with the healthy state fused, the active role of healthy state can be enhanced, while the otherness between healthy state and unhealthy state can be also activated once a fault happened. Second, manifold learning is later employed to further extract the discriminative characteristics, so that a special feature clustering distribution of reference model can be captured at each moment. Subsequently, the health performance degradation can be represented by the feature clustering distribution variation. With the merits of good class-separable clustering measure of this reference model, FCI is calculated to evaluate the feature clustering distribution. Simultaneously, an SGMbased FCC is later used to reach an accurate PDA. The 
experiments illustrates that the proposed FCC can provide a monotonic degradation profile and obviously reveal a phased degradation, which well depicts a comprehensibility of the real-world bearing PDA. In addition, an early failure warning can be sent by the significant and sharp change point arising, which exposes the occurrence of fault. Compared with other seven typical HIs, the calculated FCI has relatively higher and clearer transformation than the others. Meanwhile, compared to [7], the trigger values provided by the proposed FCC are much earlier with an effect for accurate detection. These excellent results further indicate that the SGM-based FCC is an applicable way to assess the rolling bearing health PDA.

It can be known that the proposed FCC mainly addresses evaluating the feature clustering distribution between the reference state (healthy data) and the monitor data for bearings under the given radial load. The feature clustering analysis based on the reference model can well characterize the bearing performance degradation. Nevertheless, due to the variety of working conditions in the real world, including radial loads and background environment, the insensitive feature extraction is critical to this reference model for feature clustering. To further improve the practicality of the proposed reference model, the next study is to build an experimental system and obtain the insensitive features for feature clustering distribution regardless of different loads and background noise. For an extension of the FCC, the reference model can be further implemented in bearing PDA of other key machine components and residual useful life prediction.

\section{Data Availability}

The data used in this paper have been provdied by IMS, NASA Ames Prognostics Data Repository, University of Cincinnati, NASA, and the website for the data has been listed in [28].

\section{Conflicts of Interest}

The authors declare that they have no conflicts of interest.

\section{Acknowledgments}

This work was supported by the National Natural Science Foundation of China under Grant no. 51805051 and in part by the Chongqing Research Program of Basic Research and Frontier Technology (no. cstc2019jcyj-msxmX0346) and Chongqing Special Subsidies for Post-Doctoral Research Projects (no. XmT2018038). The authors would like to thank Case Western Reserve University for offering free downloading of the bearing data.

\section{References}

[1] M. Buzzoni, E. Soave, G. D’Elia, E. Mucchi, and G. Dalpiaz, "Development of an indicator for the assessment of damage level in rolling element bearings based on blind deconvolution methods," Shock and Vibration, vol. 2018, Article ID 5384358, 13 pages, 2018.
[2] N. Trapani, M. Macchi, and L. Fumagalli, "Risk driven engineering of prognostics and health management systems in manufacturing," IFAC-PapersOnLine, vol. 48, no. 3, pp. 995-1000, 2015.

[3] X. Tang, X. Hu, W. Yang, H. Yu, and H. Yu, "Novel torsional vibration modeling and assessment of a power-split hybrid electric vehicle equipped with a dual-mass flywheel," IEEE Transactions on Vehicular Technology, vol. 67, no. 3, pp. 1990-2000, 2018.

[4] P. Tiwari and S. Upadhyay, "Degradation assessment of ball bearings utilizing curvilinear component analysis," Proceedings of the Institution of Mechanical Engineers, Part K: Journal of Multi-Body Dynamics, vol. 233, no. 3, pp. 714-730, 2019.

[5] S. Wang, J. Chen, H. Wang, and D. Zhang, "Degradation evaluation of slewing bearing using HMM and improved GRU,” Measurement, vol. 146, pp. 385-395, 2019.

[6] J. Yu, M. Liu, and H. Wu, "Local preserving projections-based feature selection and gaussian mixture model for machine health assessment," Proceedings of the Institution of $\mathrm{Me}$ chanical Engineers, Part C: Journal of Mechanical Engineering Science, vol. 225, no. 7, pp. 1703-1717, 2011.

[7] J. Yu, "Bearing performance degradation assessment using locality preserving projections and Gaussian mixture models," Mechanical Systems and Signal Processing, vol. 25, no. 7, pp. 2573-2588, 2011.

[8] F. Liu, B. He, Y. Liu, S. Lu, Y. Zhao, and J. Zhao, "Phase space similarity as a signature for rolling bearing fault diagnosis and remaining useful life estimation," Shock and Vibration, vol. 2016, Article ID 5341970, 12 pages, 2016.

[9] J. Gai, Y. Hu, and J. Shen, "A bearing performance degradation modeling method based on emd-svd and fuzzy neural network," Shock and Vibration, vol. 2019, Article ID 5738465, 10 pages, 2019.

[10] A. Rai and S. H. Upadhyay, "Bearing performance degradation assessment based on a combination of empirical mode decomposition and k-medoids clustering," Mechanical Systems and Signal Processing, vol. 93, pp. 16-29, 2017.

[11] M. Cerrada, R.-V. Sánchez, C. Li et al., "A review on datadriven fault severity assessment in rolling bearings," $\mathrm{Me}$ chanical Systems and Signal Processing, vol. 99, pp. 169-196, 2018.

[12] D. Wang, K.-L. Tsui, and Q. Miao, "Prognostics and health management: a review of vibration based bearing and gear health indicators," IEEE Access, vol. 6, pp. 665-676, 2018.

[13] T. W. Rauber, F. de Assis Boldt, and F. M. Varejão, "Heterogeneous feature models and feature selection applied to bearing fault diagnosis," IEEE Transactions on Industrial Electronics, vol. 62, no. 1, pp. 637-646, 2015.

[14] Q. He, X. Ding, and Y. Pan, "Machine fault classification based on local discriminant bases and locality preserving projections," Mathematical Problems in Engineering, vol. 2014, Article ID 923424, 12 pages, 2014.

[15] A. Malhi and R. X. Gao, "PCA-based feature selection scheme for machine defect classification," IEEE Transactions on Instrumentation and Measurement, vol. 53, no. 6, pp. 1517-1525, 2004.

[16] J. Harmouche, C. Delpha, and D. Diallo, "Linear discriminant analysis for the discrimination of faults in bearing balls by using spectral features," in Proceedings of the 2014 First International Conference on Green Energy ICGE 2014, pp. 182-187, IEEE, Sfax, Tunisia, March 2014.

[17] X. Ding, Q. He, and N. Luo, "A fusion feature and its improvement based on locality preserving projections for rolling element bearing fault classification," Journal of Sound and Vibration, vol. 335, pp. 367-383, 2015. 
[18] X. Ding and Q. He, "Two-class model based on nonlinear manifold learning for bearing health monitoring," in Proceedings of the 2016 IEEE International Instrumentation and Measurement Technology, IEEE, Taipei, Taiwan, pp. 1-6, May 2016.

[19] Y. Lu, Q. Li, and S. Y. Liang, "Physics-based intelligent prognosis for rolling bearing with fault feature extraction," International Journal of Advanced Manufacturing Technology, vol. 97, no. 1-4, pp. 611-620, 2018.

[20] X. Jin, M. Zhao, T. W. S. Chow, and M. Pecht, "Motor bearing fault diagnosis using trace ratio linear discriminant analysis," IEEE Transactions on Industrial Electronics, vol. 61, no. 5, pp. 2441-2451, 2014.

[21] F. Li, B. Tang, and R. Yang, "Rotating machine fault diagnosis using dimension reduction with linear local tangent space alignment," Measurement, vol. 46, no. 8, pp. 2525-2539, 2013.

[22] A. A. Jaber and R. Bicker, "Fault diagnosis of industrial robot bearings based on discrete wavelet transform and artificial neural network," International Journal of Prognostics and Health Management, vol. 7, p. 17, 2016.

[23] S. T. Roweis and L. K. Saul, "Nonlinear dimensionality reduction by locally linear embedding," Science, vol. 290, no. 5500, pp. 2323-2326, 2000.

[24] Q. He, "Vibration signal classification by wavelet packet energy flow manifold learning," Journal of Sound and Vibration, vol. 332, no. 7, pp. 1881-1894, 2013.

[25] J. M. Lucas and M. S. Saccucci, "Exponentially weighted moving average control schemes: properties and enhancements," Technometrics, vol. 32, no. 1, pp. 1-12, 1990.

[26] E. B. Martin and A. J. Morris, "Non-parametric confidence bounds for process performance monitoring charts," Journal of Process Control, vol. 6, no. 6, pp. 349-358, 1996.

[27] J. A. Carino, D. Zurita, M. Delgado, and R. J. Romero, "Remaining useful life estimation of ball bearings by means of monotonic score calibration," in Proceedings of the 2015 IEEE International Conference on Industrial Technology (ICIT), pp. 1752-1758, IEEE, Seville, Spain, March 2015.

[28] J. Lee, H. Qiu, G. Yu, J. Lin, and Rexnord Technical Services, Bearing Data Set, IMS, NASA Ames Prognostics Data Repository, Moffett Field, CA, USA, 2007, http://ti.arc.nasa.gov/ project/prognostic data-repository. 